\title{
A Pixel-wise Object Tracking Algorithm with Target and Background Sample
}

\author{
Chunsheng Hua Haiyuan Wu Qian Chen Toshikazu Wada \\ Faculty of System Engineering, Wakayama University, Sakaedani 930, Wakayama City, 6408510, Japan \\ Email:\{hua,wuhy,chen\}@vrl.sys.wakayama-u.ac.jp, twada@ieee.org
}

\begin{abstract}
In this paper, we present a clustering-based tracking algorithm for non-rigid object. Non-rigid object tracking is a challenging task because the target often appears as a concave shape or an object with apertures. In such cases, many background areas will be mixed into the tracking target, which are difficult to be removed by modifying the shape of the search area. Our algorithm realizes robust tracking for such objects by classifying the pixels in the search area into "target" and "background" with K-means clustering algorithm that uses both the "positive" and "negative" samples. The contributions of this research are: 1) Using a 5D feature vector to describe both the geometric feature " $(x, y)$ " and color feature " $(Y, U, V)$ " of an object (or a pixel) uniformly. This description enables the simultaneous adaptation of both the geometric and color variance during tracking; 2) Using a variable ellipse model (a) to describe the search area; (b) to model the surrounding background. This guarantees the stable tracking of objects with various geometric transformations. Through extensive experiments in various environments and conditions, the effectiveness and the efficiency of the proposed algorithm is confirmed.
\end{abstract}

\section{Introduction}

It is a challenging issue to achieve robust object tracking in unconstrained environments such as complex non-rigid movements, illumination variation, mass-clustered background, occlusion, etc. In this paper we describe a general purpose algorithm for robust object tracking which can work robustly with limited knowledge and input information.

Since our algorithm performs the pixel-wise clustering and represents the tracking result with a set of pixels, the phenomenon of template pollution caused by background interfusion, which is one of the serious problems that many conventional tracking methods suffer from, will not occur. This enables robust tracking for objects having complex shapes. Also, without the botheration of background in- terfusion, both the target model and the background model can be updated safely during tracking, which enables robust object tracking even when strong color drift of the target object occurs and/or the background varies fast.

In the last two decades, numerous powerful algorithms for object tracking were developed. They can be categorized according to 1) object representation, 2) search strategy, and 3) similarity (dissimilarity) measurement.

Object representations can be classified into four types: 1-a) object appearance [4, 5], 1-b) color histogram [6], 1-c) image features [2, 3], and 1-d) contours [12, 15]. Appearance based object representation and color histogram representation have to define a region surrounding the tracking object. Within this surrounding region, background pixels may be involved, and it sometimes causes tracking failure. Contour based object representation as well as feature based representation (such as, interest points and line segments) is not suitable for blurred and noisy image sequences and the initialization may be complex.

Search strategies can be classified into three types: 2a) brute force search within a search area [2, 3, 4, 5], 2-b) steepest ascent (descent) search [6], and 2-c) random sampling search $[8,9]$. Brute force search is time consuming and can fail when a similar object exists in the search area. Steepest ascent (descent) search performs good tracking when a smoothly distributed dissimilarity (similarity) distribution can be used. However, over smoothed distribution sometimes removes local maxima (minima) and it causes tracking failure when similar objects are located in close proximity.

Random sampling search [7] is performed in the model parameter space and has a good property that it sometimes recovers from slight tracking failure, because it maintains the model parameter distribution.

The similarity measure spreads in wide spectrum depending on the object representation. In the case of appearance model, i.e., template matching, dissimilarity measures SAD (sum of absolute difference) or SSD (sum of squared difference) are widely used. Bhattacharyya distance [10] and Kullback-Lieber divergence [11] and Jeffly divergence can be used for the dissimilarity measure between color his- 
tograms. In the case of image feature and contour based representations, Chamfer distance is a good candidate, but there are many other measures.

There are also tracking algorithms based on background subtraction [1]. Since this kind of methods assumed still background or known 3-d structure and motion of the background, they can not be used when the background is in unknown motion or has multiple moving objects.

\section{Key Ideas}

To realize a general tracking algorithm, the following situation should be considered: 1) the object shape could be complex or deformable; 2) the object's color may vary while tracking due to illumination variance. To track objects in such conditions:

1. We use the pixel-wise K-means clustering algorithm [14] to track object, because compared with the model-based methods, pixel-wise method: 1) Does not need the predefined target appearance model; 2) Can track the non-rigid or wired object well.

2. We introduce the concept of negative background into the pixel-wise method. That is because most of the existing methods only use the positive sample and a threshold to classify if an unknown pixel is the target or not. However, since a threshold is a fixed standard, those methods are not flexible enough to deal with the target/background changes. Therefore, by using background information as well as target information, the pixel classification can be performed by checking if a pixel is more similar to the target than to the background. This makes our method flexible to cope with the target/background changes.

Another motivation to introduce the background information is to solve the template pollution caused by background pixels in the target area, from which many existing tracking algorithms suffer (here, we call it as the background interfusion problem). To resolve the background interfusion problem, it is important to make the estimation between those pixels and the background samples.

In our work, an unknown pixel is classified by examining its similarity to the target and its dissimilarity to the background. As shown in Fig.1, we take pixels on the outline of the search area (in this paper, it is the ellipse contour) as the representative background samples. The pixels within the search area will be discriminated into target and background groups by checking its similarity to the target samples and its dissimilarity to the background samples. This process equals to define a discriminative plane (shown in Fig.1) and use it to perform the classification. Since this discriminative plane is defined according to the target and background samples (which are updated in each frame), no fixed threshold is needed and the background interfusion problem is also solved.

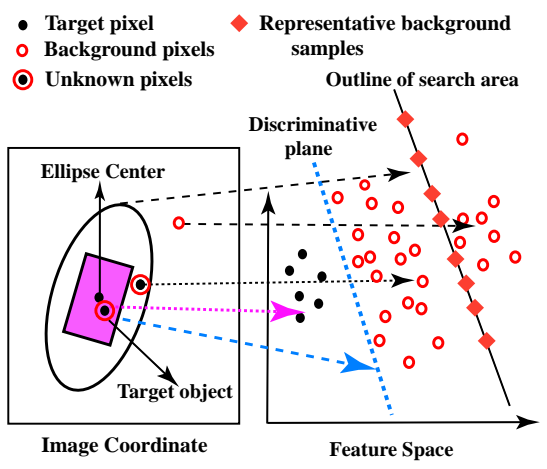

\section{Figure 1. Illustration for Background samples}

3. Using a 5D vector to represent the target/background features. In this algorithm, each pixel is described by a $5 \mathrm{D}$ feature vector $\mathbf{f}=\left[\begin{array}{ll}\mathbf{c} & \mathbf{p}\end{array}\right]^{T}$, where, $\mathbf{c}=\left[\begin{array}{lll}Y & U\end{array}\right]^{T}$ describes the color and $\mathbf{p}=\left[\begin{array}{ll}x & y\end{array}\right]^{T}$ describes the position proximity. By applying K-means clustering to both the target and background sample in 5D feature space, the target center is processed and updated not only in 2D position space but also in the $3 \mathrm{D}$ color space simultaneously. This nature guarantees our method to be robust against the illumination changes.

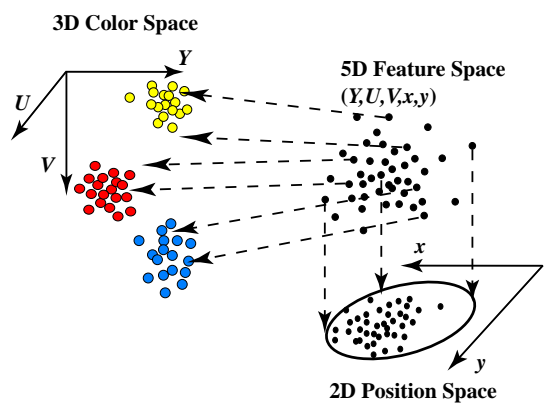

Figure 2. Illustration for 5D feature space

\section{Tracking Algorithm}

\subsection{K-means Clustering with Target and Background Samples}

As Fig.3, we use the ellipse to describe the target shape, the ellipse center to represent the target center. We also define the ellipse contour is composed of the representative background samples and all the target pixels are included in the ellipse area.

In the feature space, we describe the ellipse center as $\mathbf{f}_{T}(i)=\left[\mathbf{c}_{T}(i) \mathbf{p}_{T}(i)\right]^{T} i=1 \sim K, K$ is the number of the 
target color. Background pixels on the ellipse are presented as $\mathbf{f}_{N}(j)=\left[\mathbf{c}_{N}(j) \mathbf{p}_{N}(j)\right]^{T}, j=1 \sim m$, where $m$ is the number of the selected pixels on the ellipse, and an unknown pixel is described by $\mathbf{f}_{u}=\left[\begin{array}{ll}\mathbf{c}_{u} & \mathbf{p}_{u}\end{array}\right]^{T}$.

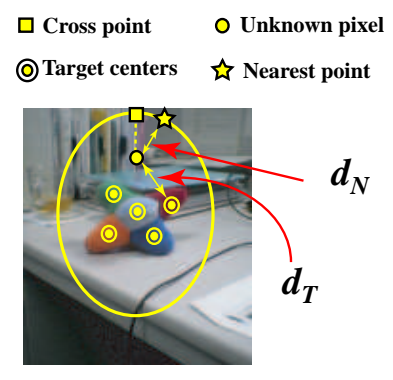

Figure 3. Illustration for target clustering

The $\mathbf{f}_{u}$ can be classified by comparing the distance $d_{T}$.

$$
d_{T}=\min _{i=1 \sim K}\left\|\mathbf{f}_{T}(i)-\mathbf{f}_{u}\right\|^{2}
$$

and the minimum distance $d_{N}$.

$$
d_{N}=\min _{j=1 \sim m}\left\|\mathbf{f}_{N}(j)-\mathbf{f}_{u}\right\|^{2}
$$

If $d_{T}<d_{N}, \mathbf{f}_{u}$ is classified as a target pixel, otherwise a background pixel. In Eq.(2), there are $m$ background points selected from the outline of search area. Here, we let $m=9$ and 8 of them are resolved by the 8 -equal division of the ellipse contour at the interval of $45^{\circ}$. The rest one is the cross point shown in Fig.3.

\subsection{Search Area Update}

After the K-means clustering, we update the search area according to the target detection result in frame $t$. To determine the shape of search area, we apply the Gaussian probability density function $(p d f)$ to represent the distribution of the detected target pixels. Since Gaussian $p d f$ is a statistical representation, it has the following advantages in describe the target appearance: 1) It can follow the deformations or the geometric transformations of the target; 2) It is insensitive to the detection errors due to image noise, etc.

In this algorithm, the Gaussian $p d f$ of a target object described by a set of vectors $\mathbf{Z}=\left[Z_{1}, Z_{2} \ldots . . Z n\right]^{T}$ can be described as:

$$
\mathbf{Z} \sim \mathcal{N}\left(\mathbf{m}_{Z}, \Sigma_{Z}\right)
$$

where $Z_{i}=\left\{x_{i}, y_{i}\right\}^{T},\left(x_{i}, y_{i}\right)$ represent the detected target pixels, $\mathbf{m}_{Z}$ is the mean and $\Sigma_{Z}$ denotes the covariance matrix. The Mahalanobis distance of a vector $\mathbf{Z}$ to the mean $\mathbf{m}_{Z}$ is given by

$$
g(\mathbf{Z})=\left[\mathbf{Z}-\mathbf{m}_{Z}\right]^{T} \Sigma_{Z}{ }^{-1}\left[\mathbf{Z}-\mathbf{m}_{Z}\right] .
$$

The minimum ellipse $(E(M))$ that contains at least $M \%$ of the target pixels is given by

$$
g(\mathbf{Z})=J
$$

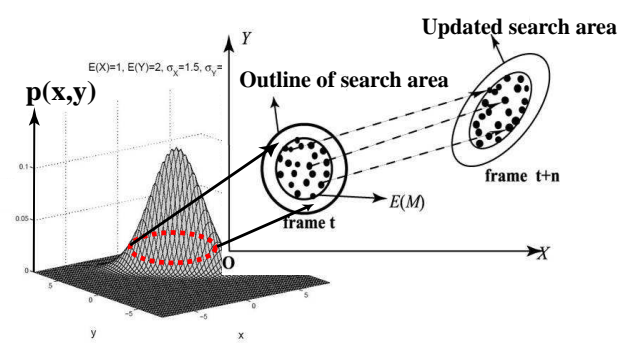

Figure 4. Update of search area.

where $J=-2 \ln \left(1-\frac{M}{100}\right)$. We let $M$ be big enough (e.g. 95) so that $E(M)$ will contain most target pixels. The outline of search area is obtained by enlarging $E(M)$ by $k$ (e.g. 1.25) times.

\section{Experiment and Discussion}

To evaluate the performance of our algorithm, we applied it to many image sequences under various conditions. Some representative experiment resluts are shown in Fig.5.

In Fig. 5 Row $1^{1}$, the challenging tasks include: 1) Low image color saturation and the color difference between the target and background is small; 2) Color and shape variance of the target was distinguished. Even the color difference is small, the target could still be segmented correctly with our $\mathrm{K}$-means clustering algorithm in the 5D feature space. Because the target information was continuously updated by the K-means algorithm, our K-means tracker had the ability to follow the rapid color shift caused by the illumination changes (frame 35,90). By updating the search area according to the distribution of the detected target pixels, our method could cope with the various target deformations and scaling changes successfully.

In Fig. 5 Row 2, the target is a monochromatic comb and the challenging issues include: 1) many background parts were mixed into the search area through the space between the teeth of the comb; 2) rapid color shift caused by the changes of the surface orientation of the target. Since our method could segment the target correctly (frame 175), the target color information could be updated safely and correctly. Because of this, even when strong color shift occurred (frame 387), our algorithm could still catch the target successfully. Although the background with similar color

\footnotetext{
${ }^{1}$ PETS2001 public database, the University of Reading, UK, http://peipa.essex.ac.uk/ipa/pix/pets/PETS2001
} 

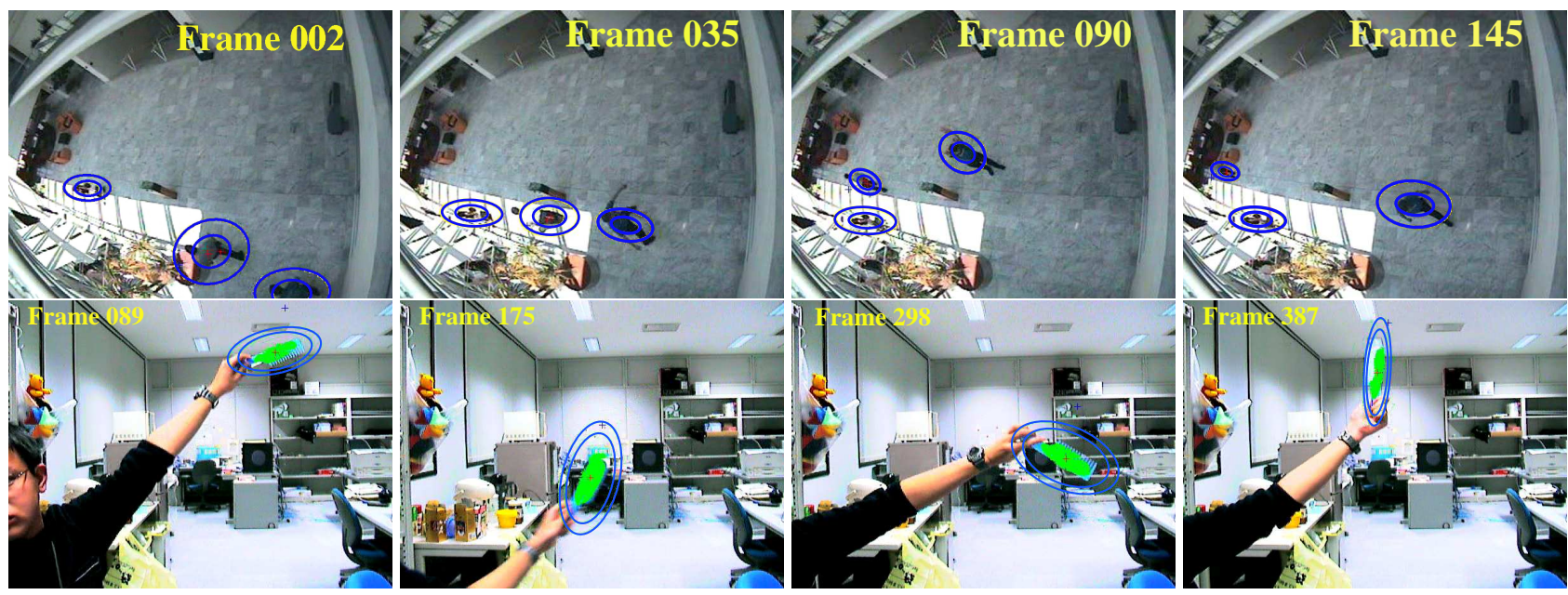

Figure 5. Experiment results with different target under various conditions

was mixed into the search area in frame 175 , it was removed successfully from the target cluster because of the distinct difference between it and the target in the 5D feature space.

All the experiments were taken with a desktop PC with 3.06GH Intel XEON CPU, and the image size was $640 \times$ 480 pixels. When the target size varied from $140 \times 140$ $\sim 200 \times 200$ pixels, the processing speed of our algorithm was about $12 \sim 18 \mathrm{~ms} /$ frame.

We also compared our algorithm with the Mean shift and the SAD template matching algorithms. Some of the experiment results are available at http://www.wakayamau.ac.jp//wuhy/wu2_new.html.

\section{Conclusion}

In this paper, we proposed a general object tracking algorithm. With the pixel-wise clustering method, our algorithm achieved the robust tracking with little prior knowledge and assumption. By applying the K-means clustering algorithm to both the target and background samples in the 5D feature space that describes the color and geometric information uniformly, our method can discriminate the target from the surrounding and interfused background pixels successfully. Based on this algorithm, we realized a real video-rate tracking system.

Acknowledge: This research is partially supported by the Ministry of Education, Culture, Sports, Science and Technology, Grantin-Aid for Scientific Research (A)(2), 16200014 and (C)18500131, (C) (2) 15500112.

\section{References}

[1] Stauffer, C. and Grimson, W.E.L, Adaptive Background Mixture Models for Real-time Tracking, CVPR, pp.246-252 1999
[2] R. Collins and Y. Liu, On-line Selection of Discriminative Tracking Feature, ICCV, Vol.1 pp.346-352 2003

[3] H.T.Nguyen and A. Semeulders, Tracking aspects of the foreground against the background, ECCV, Vol.2 pp.446-456 2004

[4] H.D.Crane and C.M.Steele, Translation-tolerant mask matching using noncoherent reffective optics, PR, Vol.1, No.2, pp.129-136, 1968

[5] C.Grä $\beta 1$, T.Zin $\beta$ er, H.Niemann, Illumination Insensitive Template Matching with Hyperplanes, DAGM, pp.273-280, 2003

[6] D.Comaniciu, V.Ramesh and P.Meer, Kernel-Based Object Tracking, PAMI, Vol.25, No.5, pp.564-577, 2003

[7] M.Isard, A.Blake, Condensation-conditional density propagation for visual tracking, IJCV, Vol.29, No.1, pp.5-28, 1998

[8] J. Deutscher, B. North, B. Bascle and A. Blake, Tracking through singularities and discontinuities by random sampling Proc. ICCV, pp.1144-1149, 1999.

[9] K.Smith and D. Gatica-Perez, Order matters: A Distributed Sampling Method for Multi-Object Tracking, BMVC 2004

[10] Fred C. Schweppe. State space evaluation of the Bhattacharyya distance between two Gaussian processes. Information and Control, 11(3):352-372, September 1967.

[11] HZ.Rafi and H.Soltanianzadeh, Mutual Information Restoration of Multispectral Images, Proc.IWSSIP, 2003. 2

[12] M.Kass, A. Witkin and D. Terzopoulos, Snakes: active contour models, IJCV, Vol.1, pp.321-332, 1988

[13] B.Heisele, U,Kre $\beta$ el, W.Ritter, Tracking Non-Rigid Moving Objects Based on Color Cluster Flow, CVPR, pp.253-257 1997

[14] J.Hartigan, M.Wong, Algorithm AS136: A K-Means Clustering Algorithm, Applied Statistics, Vol.28, pp.100-108, 1979

[15] M.Isard, A.Blake, Contour tracking by stochastic propagation of conditional density, ECCV, Vol.1, pp.343-356, 1996 